\title{
Flexible Magnetoresistive Sensors for Guiding Cardiac Catheters
}

\author{
M. Hawsawi ${ }^{1}$, S. Amara ${ }^{1}$, Y. Mashraei ${ }^{1}$, A. Almansouri ${ }^{1}$, H. Mohammad ${ }^{1}$, G. Torres Sevilla ${ }^{1}$, G. Jakob ${ }^{2}$, S. Jaiswal ${ }^{2,3}$, \\ M. Kläui ${ }^{2}$, A. Haneef ${ }^{4}$, A. Saoudi ${ }^{4}$, M. Hussain ${ }^{1}$ and J. Kosel ${ }^{1}$ \\ ${ }^{1}$ Computer, Electrical and Mathematical Science and Engineering Division, King Abdullah University of Science and \\ Technology, Thuwal 23955, Saudi Arabia \\ ${ }^{2}$ Institut für Physik, Johannes Gutenberg Universität Mainz, Mainz 55128, Germany \\ ${ }^{3}$ Singulus Technologies AG, 63796 Kahl am Main, Germany. \\ ${ }^{4}$ King Abdullah International Medical Research Center, Riyadh 14611, Saudi Arabia \\ jurgen.kosel@kaust.edu.sa
}

\begin{abstract}
Cardiac catheterization is a procedure, in which a long thin tube that is called a "catheter" is inserted into the heart for diagnosis or treatment. Due to the excessive use of $x$-ray doses and contrast agents for orientation detection during the surgery, there is a need to find a better alternative. This paper presents magnetic tunnel junction sensors on flexible $\mathrm{Si}$ attached to the catheter tip for orientation detection during minimally invasive surgeries. Due to the small size of catheters, extreme minimization in terms of size, weight, thickness and power consumption is needed for any device implemented on it. The fabricated flexible magnetic tunnel junctions fulfill those requirements with size, thickness, weight and power consumption of $150 \mu \mathrm{m}^{2}, 12 \mu \mathrm{m}, 8 \mu \mathrm{g}$ and $0.15 \mu \mathrm{W}$, respectively, while still providing a high sensitivity of $4.93 \%$ /Oe. The sensors can be bent with up to $500 \mu \mathrm{m}$ in diameter, which is more than needed for even the smallest catheters of size $1 \mathrm{~mm}(3 \mathrm{Fr})$ in diameter. This result is a stepping-stone towards the development of a versatile and low-cost smart catheter system that can help surgeons navigate inside the heart while minimizing the side effects.
\end{abstract}

Keywords-Magnetic Tunnel Junctions; Tunnel Magnetoresistive Sensor; Flexible Sensor; Orientation; Catheter

\section{INTRODUCTION}

Cardiac catheterization is a minimally invasive surgery that is performed to diagnose or treat cardiovascular conditions. During the surgery, a catheter is threaded to the heart from a vein in the groin, neck or arm. The catheter helps the cardiac surgeon in diagnosing the heart by locating any blockage in the blood vessels, obtaining a tissue sample from the heart or checking the pumping function of the heart. Also, the catheter can be used for treating the heart during procedures such as angioplasty, closure of holes, replacement of heart valves, and ablation. The common method for visualizing the heart during the surgery uses fluoroscopy, which requires the use of contrast agents and $\mathrm{x}$-ray imaging in order to confirm the position and orientation of the catheter tip as well as check the blood flow in the coronary arteries [1]. Thus, a continuous x-ray beam must be passed through the body of the patient to provide the surgeon with a visual image of the heart and the catheter that is presented on a monitor (Fig. 1).
While cardiac catheterization is minimally invasive and generally considered safe, compared to open surgery, there exist serious side effects that may affect the patients. One of the major limitations is the use of x-ray and contrast agents when determining the position and orientation of the catheter. A series of attempts to get into the targeted vessel branch and put the catheter into the intended position and orientation is often required, leading to a series of contrast dye injections and extended $\mathrm{x}$-ray exposure. It is important to note that one of the common causes of acute rental dysfunction is the contrast medium-induced nephropathy (CIN), which has gained increased attention in clinical settings, especially during a cardiac intervention. The occurrence of CIN is reported to be $2 \%$ in the general population; however, the percentage could rise to $20 \%-30 \%$ in high-risk patients (i.e. patients with chronic renal impairment, diabetes mellitus, congestive heart failure, and older age). Higher contrast volume is a serious risk factor for CIN [2]. Another concern is the long X-ray exposure, which causes radiation side effects to both the patients and medical staff.

A recently developed technology that aims to decrease the $\mathrm{x}$-ray exposure during cardiac catheterization is the remote magnetic navigation system. It consists of two focused-field permanent magnets inside of a housing, which are positioned on each side of the patient's body. The two magnets create a relatively uniform magnetic field of approximately $0.08 \mathrm{~T}$, which can penetrate $15 \mathrm{~cm}$ inside the chest of the patient. A small permanent magnet is placed on the tip of the catheter, which aligns itself with the applied magnetic field produced by the magnets outside of the body. If the orientations of the outer magnets with respect to each other change, the magnetic field changes accordingly and, as a consequence, the catheter tip gets deflected. Thereby, the surgeon can navigate the catheter via a computer-controlled system without any manual manipulation [3][4]. However, this method requires special equipment and installation, making it a bulky and a very expensive solution. In addition, it is not compatible with the force measurement tools that are currently being used in hospitals and does not provide position or orientation feedback. 

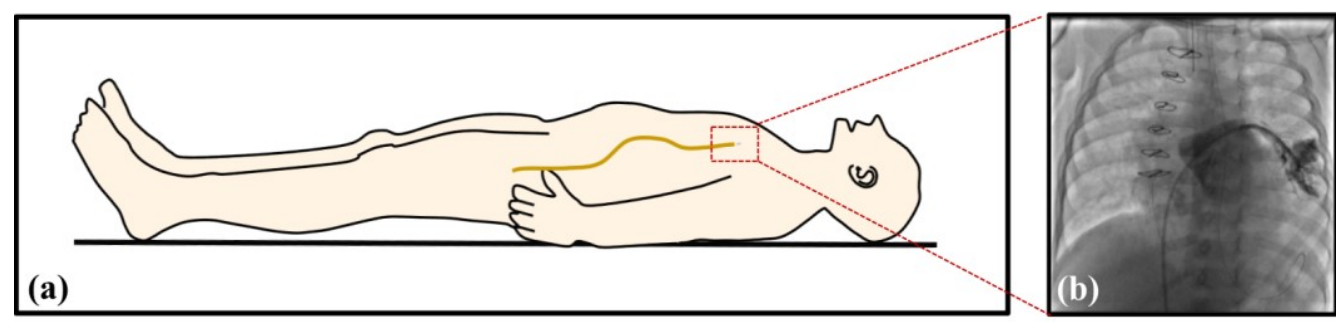

Fig. 1. (a) A schematic of a patient that has a cardiac catheter inserted through the groin. (b) An x-ray image showing the catheter and the contrast dye is spread in the arteries [5].

A 3-axis magneto-impedance sensor system has also been proposed as a navigation tool to detect the position and orientation of a catheter tip to minimize the $\mathrm{x}$-ray exposure during cardiac catheterization. The system exploits the earth magnetic field together with an $\mathrm{AC}$ magnetic field of $10 \mathrm{kHz}$ that is produced by a two-axis magnetic field coil. A 3-axis magneto-impedance effect sensor is used to detect both the Earth magnetic field and the AC magnetic field. Two Euler angles are obtained from the measurements of the Earth magnetic field while the $\mathrm{AC}$ magnetic field measurements provide the third Euler angle, representing the orientation of the tip. Even though this system offers a navigation system that potentially minimizes x-ray exposure, it requires the detection of two different magnetic fields, making the system complicated [6]. Magneto-impedance sensors are a good choice for their high sensitivity, but they operate at high frequencies (around $100 \mathrm{MHz}$ to $\mathrm{GHz}$ ), which requires complex electronics, and which makes them very sensitive to changes in their environment (i.e. different tissues types etc.). Most importantly, magneto-impedance sensors are relatively bulky. The size of the sensor used was $2 \mathrm{~mm} \times 2 \mathrm{~mm} \times 3 \mathrm{~mm}$, which is very large compared to sizes of catheter devices, and there is little potential for further miniaturization.

On the other hand, recent advancements in the field of flexible electronics have introduced the possibility of modifying devices to fit on curved surfaces. These technologies range from flexible light-emitting diodes to flexible printed circuit boards that are already considered an industrial standard for various biomedical applications or consumer electronics [712]. Flexible electronics are advantageous compared to rigid ones, due to the extreme thinness and conformity to curved surfaces that they can provide. Recent developments in thin film technologies have allowed for fabricating extremely sensitive, flexible, high-performance magnetic sensors based on the Giant Magnetoresistive (GMR) effect and Tunneling Magnetoresistive (TMR) effect [13-18]. Magnetic tunnel junctions (MTJs) are explored in this paper, due to their low power consumption and high sensitivity as magnetic sensors [19-21].

This paper presents the fabrication of flexible MTJs, their use as angle sensors, and their application on a cardiac catheter.
Fig 2. shows a schematic of the sensor concept, which is based on detecting the components of the Earth's magnetic field by an MTJ sensor. High performance of the MTJs is obtained by implementing them on rigid Si substrate, which is then thinned down to make it mechanically flexible. The proposed method provides a highly miniaturized sensor that can fit on the tip of any standard catheter with negligible increase in mass or size. It is characterized to have an extremely low power consumption and high sensitivity, which minimizes the complexity and costs.

\section{METHODS}

The MTJ stack was deposited onto thermally oxidized 8" Si wafers with orientation (001) using a magnetron sputtering system (Singulus Rotaris). The multilayer films of the MTJ stack are based on a PtMn exchange bias layer and a $\mathrm{CoFeB} / \mathrm{MgO} / \mathrm{CoFeB}$ tunneling structure. The sensors were patterned into elliptical shapes of $1 \mu \mathrm{m} \times 1.2 \mu \mathrm{m}$ by standard photolithography. An easy axis was induced in the free layer of the MTJ along the long axis of the ellipse, by field annealing in $8 \mathrm{kOe}$ at $360^{\circ} \mathrm{C}$ for two hours. A $7 \mu \mathrm{m}$ photoresist layer was spin-coated on the top surface of the devices acting as a protective layer in the following etching steps. Next, the MTJs were made flexible by thinning of the substrate using a softetch-back method on the $\mathrm{Si}(500 \mu \mathrm{m}) / \mathrm{SiO}_{2}(300 \mathrm{~nm})$ substrate [23][24]. Fig. 3 summarizes all the main steps of the fabrication process.

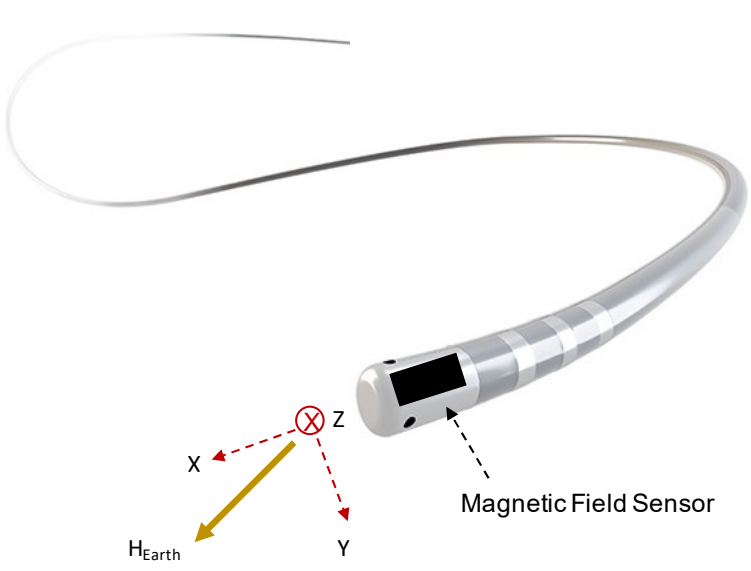

Fig. 2. A schematic of the sensor concept showing a cardiac catheter with magnetic field sensors that can measure the $x, y$, and $z$ components of the Earth magnetic field [22]. 


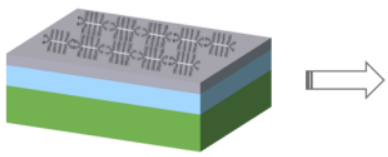

(a)

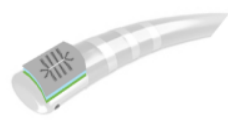

(f)

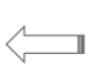

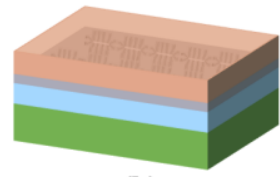

(b)

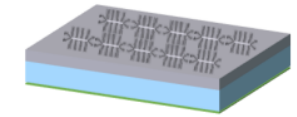

(e)

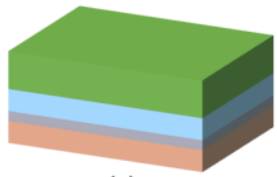

(c)

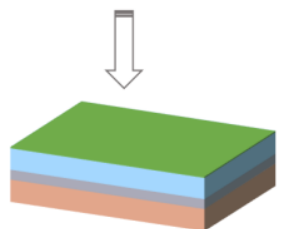

(d)

Fig. 3. The fabrication process of flexible MTJs. (a) Patterned MTJ stack on top of $\mathrm{Si} / \mathrm{SiO}_{2}$ layers. (b) Photoresist is spin-coated on top of the devices acting as a protection layer. (c) The wafer is flipped for backside etching. (d) Deep reactive ion etching of the Silicon substrate. (e) Flexible MTJ devices after the thinning process. (f) An MTJ sensor implemented on the tip of a cardiac catheter [22].

The thickness of the silicon substrate was reduced from 500 $\mu \mathrm{m}$ to $15 \mu \mathrm{m}$ by performing deep-reactive-ion-etching on the backside of the substrate. The complete process was divided into three main etching stages to produce a reliable etching process as well as protect the devices from any damages. First, $300 \mu \mathrm{m}$ were etched from the wafer. Then, the thickness was reduced by $50 \mu \mathrm{m}$ for three consecutive times until a thickness of $50 \mu \mathrm{m}$ was reached. Finally, the thickness was reduced in 5 $\mu \mathrm{m}$ steps until a final thickness of $15 \mu \mathrm{m}$ was achieved.

Fig. 4 shows the magnetoresistance measurements of the MTJ sensors. All measurements were performed at room temperature using a conventional DC four-probe method. Helmholtz coils were used to provide a homogeneous magnetic field, which was monitored using an F.W. Bell Gaussmeter model 6010 and Keithley 2400-C was used to produce a current of $10 \mathrm{~mA}$ in 4-wire resistance mode. A double-sided adhesive tape was used in the static bending experiments to fix the flexible samples on top of PMMA cylinders with different diameters ranging from $3 \mathrm{~mm}$ to $30 \mathrm{~mm}$. In addition, the resistance of the MTJ sensors was measured as a function of the direction of the magnetic field. To this end, a rotary table was designed using laser-cut, non-magnetic PMMA sheets and the sensor was implemented on top of it using a double-sided

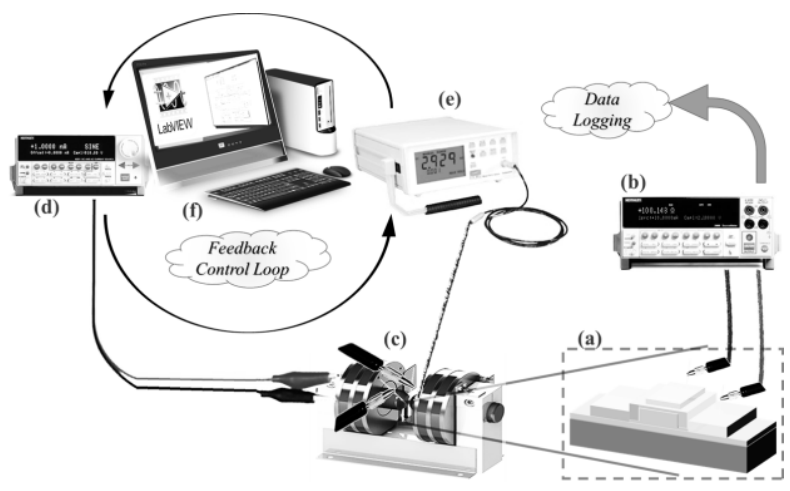

Fig.4. Measurement Setup flexible MTJ: (a) The MTJ sensor. (b) Keithley 2400-C connected in resistance measurement mode. (c) Helmholtz coils. (d) Keithley 2400-C in current-source mode. (e) F.W. Bell Gaussmeter Model 6010. (f) Computer using LabView for control and data storage adhesive tape for different angle measurements. The measurements were performed using a DC four-probe method, and the magnetic field was swept from -100 Oe to 100 Oe with 1 Oe steps.

\section{RESULTS \& DISCUSSION}

Fig.5 shows SEM images of MTJ sensors on a flexible silicon substrate thickness after the back-etching process. The thickness of the silicon substrate can be estimated to approximately $12 \mu \mathrm{m}$, which is in the desired range of $10-15$

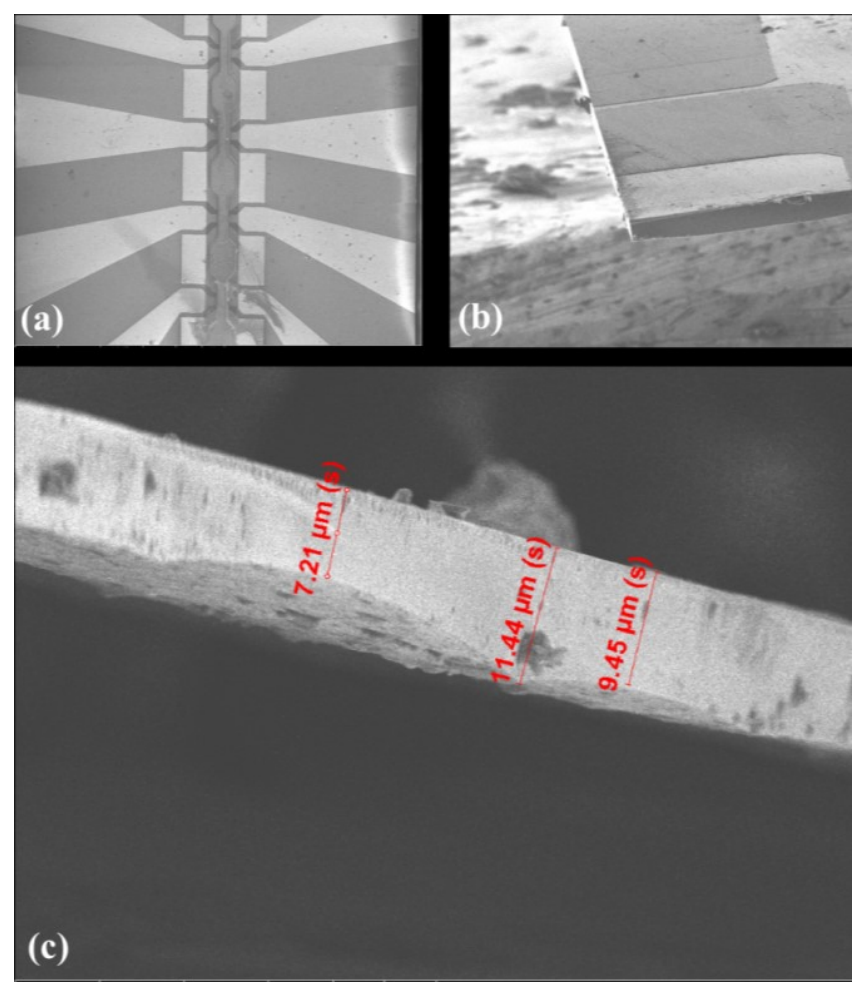

Fig.5. SEM images of the flexible MTJ sensors on a flexible silicon substrate. (a) Three MTJ sensors. (b) Side view of the silicon substrate. (c) Thickness measurements of the silicon substrate. 


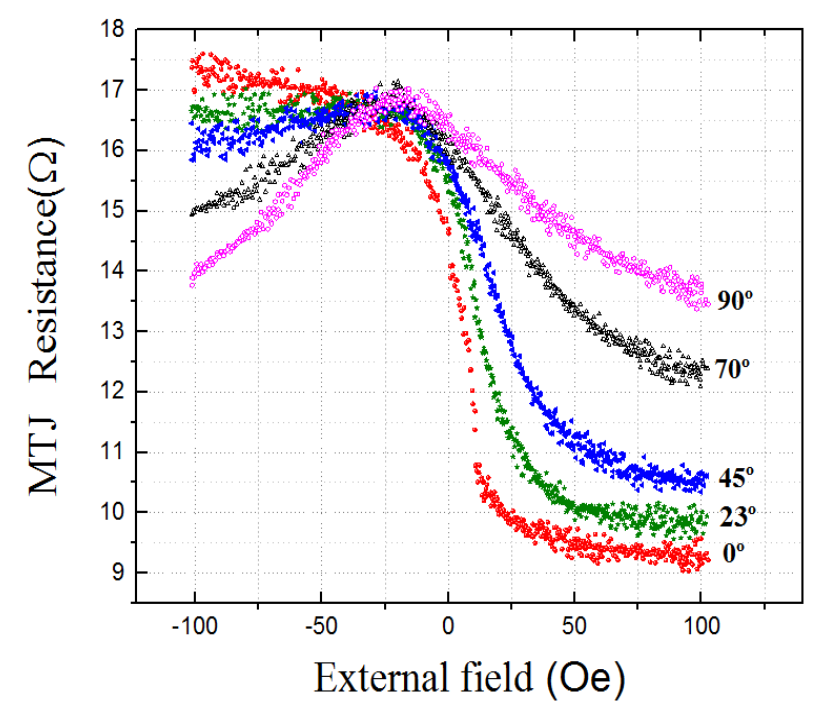

Fig. 6. Characterization curves of an MTJ sensor at five different angles.

$\mu \mathrm{m}$. By characterizing the MTJ sensors, we found that using this process maintained the magnetic properties of the MTJs and the magnetoresistance characteristics remains intact without degrading the performance in terms of tunnel magnetoresistance (TMR) ratio compared to their rigid counterpart.

Fig. 6 shows the typical resistance response of an MTJ sensor to the direction of the magnetic field. The angle was varied from $0^{\circ}, 23^{\circ}, 45^{\circ}, 70^{\circ}$ and $90^{\circ}$ with respect to the MTJ's easy axis, and the sensitivity of the MTJ changes accordingly. It can be seen that the MTJ resistance values have changed for each angle when the magnetic field was swept from -100 Oe to 100 Oe. The MTJ's has the sensitive region shifted by 20 Oe, which is caused by the influence of the pinning layer on the free sensitive layer of the MTJs. In addition, Fig 7. Summarizes the sensor response to the different angles at $0.1 \mathrm{Oe}$, which is less than the Earth magnetic field that typically ranges from 0.25 Oe to 0.65 Oe. The flexible MTJs show a responsive change in resistance in earth magnetic field when rotating,

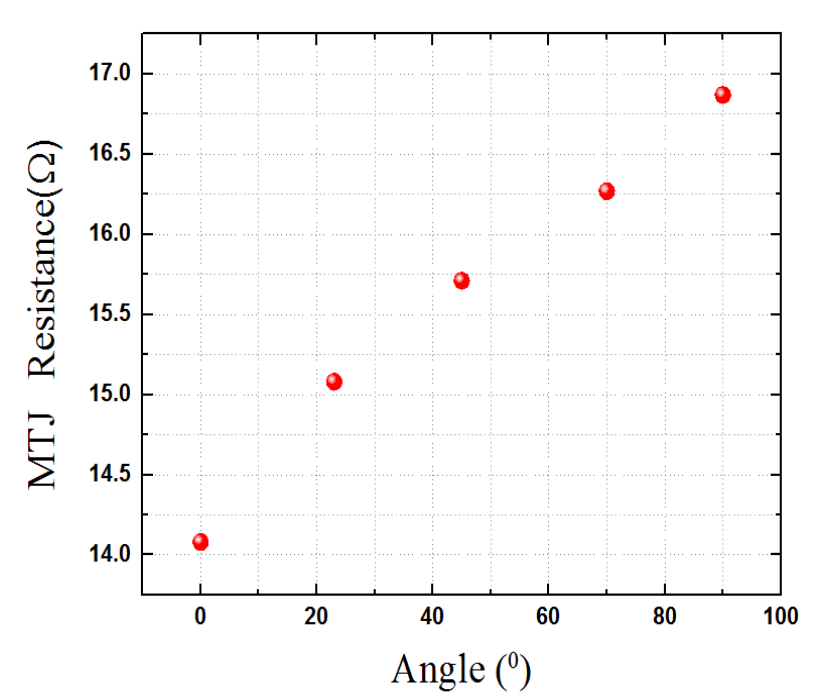

Fig. 7. Resistance of an MTJ sensor for five different angles of the applied field of $0.1 \mathrm{Oe}$.

which indicates that the flexible MTJs can indeed work as angle sensors. They also are sensitive to the Earth magnetic field, making them ideal for orientation detection. The maximum sensitivity of the MTJ sensors was $4.93 \% /$ Oe with a power consumption of $0.15 \mu \mathrm{W}$, and the weight of the sensor is a mere $8 \mu \mathrm{g}$, which was measured using a micro scale.

The ultra-thin substrate makes the fabricated MTJ films ultraflexible and suitable for conformal fitting onto the surface of catheters. Hence, the flexible MTJs were attached using superglue (LOCTITE 4011) to a diagnostic catheter (6F) for demonstration. It can be seen in Fig. 8 that the fabricated MTJ sensors are small enough to fit on the tip of a catheter with a diameter of $2 \mathrm{~mm}$. The $12 \mu \mathrm{m}$ thick sensor with a weight of 8 $\mu \mathrm{g}$ causes only a negligible change of the catheter's properties in terms of handling or size, adding approximately $0.00018 \%$ to the weight of this catheter, which is insignificant.

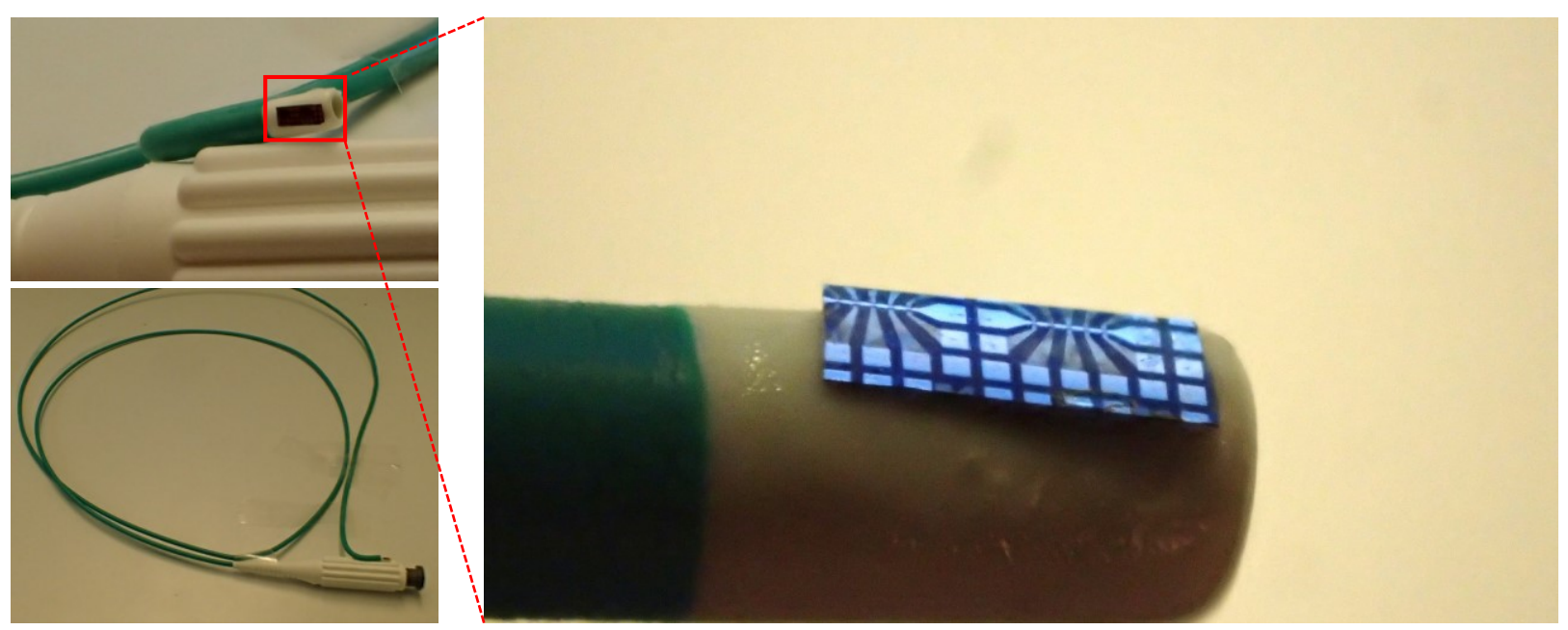

Fig. 8. The attachment of flexible MTJ sensors onto the tip of a diagnostic cardiac cartheter $(6 \mathrm{~F})$, which is $2 \mathrm{~mm}$ in diameter. 


\section{CONCLUSION}

This paper showed the fabrication process of flexible magnetic tunnel junctions for applications on cardiac catheters. With the presented fabrication method flexible devices can be fabricated using standard processes without introducing any design constraints. The magnetic properties and the magnetroresistive characteristics of the flexible MTJs remained the same as the ones of the rigid types without compromising the performance of the device.

The fabricated MTJ sensors were $15 \mu \mathrm{m}$ thick, had a power consumption of $0.15 \mu \mathrm{W}$ and a weight of $8 \mu \mathrm{g}$. They were sensitive to orientation changes, utilizing only the Earth's magnetic field, making them suitable for navigation systems. The characterization results of the sensors have shown changes in resistance values for different angles and different applied fields. Moreover, the flexible sensors were attached to the tip of a catheter, thereby only minimally adding to its size and weight, to avoid effects on handling or mechanical properties. It is worth mentioning that the developed concept can be used for different instruments and is not limited to cardiac catheters.

\section{ACKNOWLEDGMENT}

Research reported in this publication was supported by King Abdullah University of Science and Technology (KAUST).

\section{REFERENCES}

[1] R. Hotstetter et al., "Fluoroscopy As an Imaging Means for Computerassisted Surgical Navigation," Computer Aided Surgery, vol. 4(2), pp. 65-76, 1999

[2] M. Tepel, P. Aspelin and N. Lameire. "Contrast-induced Nephropathy: A clinical And Evidence-based Approach," Circulation, vol. 113(14), pp. 1799-1806, April 2006.

[3] D. Filgueiras-Rama et al., "Remote Magnetic Navigation For Accurte, Real-time Catheter Positioning and Ablation In Cardiac Electrophysiology Procedures," Journal of Visualized Experiments, vol. 74, 2013.

[4] L. Di Biase et al. "Remote Magnetic Navigation - Human Experience in Pulmonary Vein Ablation," Journal of the American College of Cardiology, 2007

[5] N. Sasikumar et al. "Congenital Pulmonary vein stenosis - Diagnosis and treatment," International Journal of Medical Research and Review. vol. 1, 2013

[6] K. Totsu, Y. Haga and M. Esashi, "Three-Axis Detecting Position And Orientation of Catheter Tip," Sensors and Actuators: Physical. vol. 111(2-3), pp. 304-309, 2004.

[7] G. Gustafsson et al., "Flexible Light-emitting Diodes Made from Soluble Conducting Polymers," Nature, vol. 357, pp. 477-479, 1992.

[8] J. A. Rogers et al., "Paper-like Electronic Displays: Large-area RubberStamped Plastic Sheets of Electronics and Microencapsulated Electrophoretic Inks," Proceedings of the National Academy of Sciences of the United States of America, vol. 98(9) , pp. 4835-4840, 2001.

[9] M. Alnassar, Y. P. Ivanov and J. Kosel, "Flexible Magnetoelectric Nanocomposites with Tunable Properties," Advanced Electronic Materials, 2016

[10] A. Alfadhel and J. Kosel, "Magnetic Nanocomposite Cilia Tactile Sensor," Advanced Materials, 27, 47, pp. 7888-7892, 2015
[11] M. Ghonheim et al., "Thin PZT Based Ferroelectric Capacitors On Flexible Silicon for Nonvolatile Memory Applications," Advanced Electronic Materials, 1500045, 10p, 2015.

[12] B. Li, M. Kavaldzhiev and J. Kosel, "Flexible Magnetoimpedance Sensor," Journal of Magnetism and Magnetic Materials, 378, pp. 499 505, 2015.

[13] M. Melzer et al., "Magnetoresistance of rolled-up Fe3Si nanomembranes," Nano Letters, vol. 11, pp. 2522, 2011.

[14] G. Lin et al., "A highly flexible and compact magnetoresistive analytic device," Lab on a chip, vol. 14, pp. 4050-4058, 2014.

[15] S. S. P. Parkin, K. P. Roche and T. Suzuki, "Giant Magnetoresistance in Antiferromagnetic $\mathrm{Co} / \mathrm{Cu}$ Multilayers Grown on Kapton," Japanese Journal of Applied Physics, 31 L1246, 1992.

[16] S. S. P. Parkin, "Flexible giant magnetoresistance sensors," Applied Physics Letters, vol. 69 , pp. 3092, 1996.

[17] X. Sun et al., "Flexible semi-transparent organic spin valve based on bathocuproine", Applied Physics Letters, vol. 105, 083302, 2014.

[18] A. Bedoya-Pinto et al., "Flexible spintronic devices on Kapton," Applied Physics Letters, vol. 104 , pp. 062412, 2014.

[19] S. S. P. Parkin et al., "Giant tunnelling magnetoresistance at room temperature with $\mathrm{MgO}$ (100) tunnel barriers," Nature Materials, vol. 3 , pp. 862-862, 2004.

[20] S. Yuasa et al., "Giant room-temperature magnetoresistance in singlecrystal Fe/MgO/Fe magnetic tunnel junctions," Nature Materials, vol. 3, pp. 868-871, 2004.

[21] M. Donolato et al., "Flexible and stretchable polymers with embedded magnetic nanostructures," Advanced Materials, 25, 623 (2013).

[22] Boston Scientific, Temperature Ablation Catheter. Available: http://www.bostonscientific.com/en-US/products/catheters-ablation/blazer-oi.html [Accessed: 3- Feb- 2018]

[23] G. Sevilla et al. "Flexible Nanoscale High-Performance FinFETs," ACS Nano, vol. 8(10), pp. 9850-9856, 2014.

[24] J. Pojas et al. "Transformational Silicon Electronics," ACS Nano, vol. $8(2)$, pp. 1468-1474, 2014 